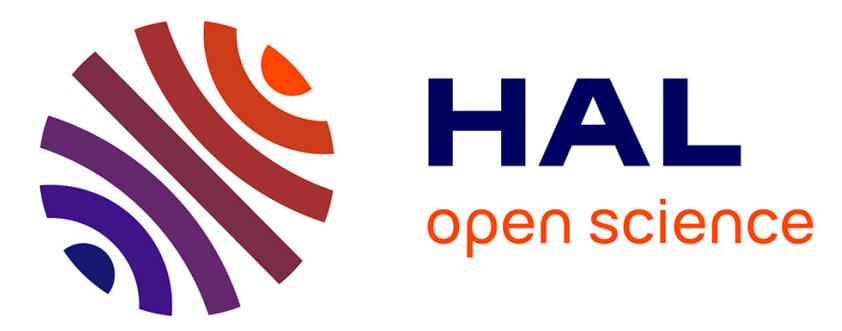

\title{
Distance-based measures of spatial concentration: Introducing a relative density function
}

\author{
Gabriel Lang, Eric Marcon, Florence Puech
}

\section{To cite this version:}

Gabriel Lang, Eric Marcon, Florence Puech. Distance-based measures of spatial concentration: Introducing a relative density function. The Annals of Regional Science, 2020, 64 (2), pp.243-265. 10.1007/s00168-019-00946-7 . hal-01082178v4

\section{HAL Id: hal-01082178 \\ https://hal.science/hal-01082178v4}

Submitted on 24 Oct 2019

HAL is a multi-disciplinary open access archive for the deposit and dissemination of scientific research documents, whether they are published or not. The documents may come from teaching and research institutions in France or abroad, or from public or private research centers.
L'archive ouverte pluridisciplinaire $\mathbf{H A L}$, est destinée au dépôt et à la diffusion de documents scientifiques de niveau recherche, publiés ou non, émanant des établissements d'enseignement et de recherche français ou étrangers, des laboratoires publics ou privés. 


\title{
Distance-Based Measures of Spatial Concentration: Introducing a Relative Density Function
}

\author{
Gabriel Lang ${ }^{1}$, Eric Marcon², Florence Puech ${ }^{3 \star}$
}

\begin{abstract} the limits of the $m$ function for detecting spatial structures in economics.

Keywords

Spatial Concentration, Aggregation, Point Patterns, Agglomeration, Economic Geography

${ }^{1}$ UMR MIA-Paris, AgroParisTech, INRA, Université Paris-Saclay, France.

${ }^{2}$ AgroParisTech, UMR EcoFoG, Cirad, CNRS, INRA, Université des Antilles, Université de Guyane, France.

${ }^{3}$ RITM, Univ. Paris-Sud, Université Paris-Saclay \& CREST, France.

*Corresponding author: florence.puech@u-psud.fr

\& Authors are in alphabetical order.
\end{abstract}

For more than a decade, distance-based methods have been widely employed and constantly improved in spatial economics. These methods are a very useful tool for accurately evaluating the spatial distribution of economic activity. We introduce a new distance-based statistical measure for evaluating the spatial concentration of industries. The $m$ function is the first relative density function to be proposed in economics. This tool supplements the typology of distance-based methods recently drawn up by Marcon and Puech (2017). By considering several simulated and real examples, we show the advantages and

\section{Contents}

\section{Introduction}

2 Approach

2.1 Basic framework . . . . . . . . . . . . 2

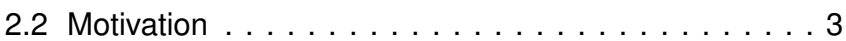

3 Definition of the $m$ function 5

3.1 An intuitive presentation . . . . . . . . . . 5

3.2 Mathematical definition $\ldots \ldots \ldots \ldots$

3.3 Properties . . . . . . . . . . . . . . . 6

3.4 Inhomogeneity or interactions $\ldots \ldots \ldots \ldots$

4 Application 7

4.1 Independent cases . . . . . . . . . . . . . 7

4.2 Aggregated cases . . . . . . . . . . . . . 7

4.3 Regular cases . . . . . . . . . . . . 9

5 Discussion

5.1 Comparisons on the three previous simulated cases . 9

5.2 Comparisons on a more complex example . . . . . . 12

6 Conclusion

\section{Introduction}

Industrial agglomerations are doubtless the main feature of today's economic geography (Krugman, 1991; Henderson and Thisse, 2004). Thus, it is not surprising that much recent research has attempted to improve the measurement of the spatial concentration of activities. ${ }^{1}$ Distance-based meth-

\footnotetext{
${ }^{1}$ See Duranton and Overman (2005); Marcon and Puech (2003, 2010); Arbia et al. (2012); Mori and Smith (2013); Jensen and Michel (2011); Bonneu and Thomas-Agnan (2015); Howard et al. (2016), among others.
}

ods are the latest statistical measures to be proposed in the field of spatial economics for detecting spatial structures (geographic concentration or dispersion). By treating space as continuous, distance-based methods provide a detailed analysis with robust results. ${ }^{2}$ Consequently, many authors consider them to be very promising techniques (Combes and Overman, 2004; Combes et al., 2008; Duranton, 2008; Arbia, 2016) and that they open the way for new explanations of the spatial concentration of activities (Ellison et al., 2010; Alfaro and Chen, 2014; Kerr and Kominers, 2015; Sweeney and GómezAntonio, 2015; Behrens and Guillain, 2017; Gómez-Antonio and Sweeney, 2018). Today, the Duranton and Overman's $K_{d}$ function (Duranton and Overman, 2005) is the most used distance-based method in economics (Sweeney and GómezAntonio, 2015; Marcon and Puech, 2017). It is a density function that evaluates absolute concentration (Marcon and Puech, 2015, 2017). In this article for the first time we shall introduce a relative density function in spatial economics, in a similar vein to the well-known location quotient (Florence, 1972). We have developed this for two main reasons. First, this new function, called $m$, supplements the typology of distance-based methods recently drawn up by Marcon and Puech (2017). Second, as Brülhart and Traeger (2005) have stressed, the nature of the spatial concentration (absolute, relative) matters. We shall prove that both $K_{d}$ and $m$ are useful for evaluating the spatial distribution of activities because of the complementary results they provide. For this, we shall give various comparisons of $K_{d}$ and $m$ results obtained with simulated and real examples to understand the advantages and

\footnotetext{
${ }^{2}$ Duranton and Overman (2008) provide many concrete examples of the problems such functions can solve.
} 
the limits of both distance-based measures.

There is growing evidence that distance-based measures are now preferred in spatial economics since Duranton and Overman's seminal paper (Duranton and Overman, 2005). One of the main reasons for this is that they preserve the richness of individual data. Unlike the Gini (1912) or the Ellison and Glaeser (1997) indices, distance-based methods do not rely on any predefined zoning (regions, counties...). Distance-based methods are implemented directly using the position of entities (stores, plants...). The analysis of the spatial distribution of entities is based on the distance between them. In contrast, it has been proven that indices that aggregate data at a zonal level are sensitive to the zoning chosen (Arbia, 2001; Briant et al., 2010) as described by the Modifiable Areal Unit Problem - MAUP (Openshaw and Taylor, 1979; Arbia, 1989). One way of solving MAUP issues is to treat space as "continuous" as suggested by Duranton (2008). This is the main feature of distance-based measures. Consequently, MAUP issues vanish because the data is treated without loss of information or modification of the raw dataset. The spatial structure of the distribution is analyzed at all scales simultaneously (and not at only one level of observation as it is the case with spatial zoning). Multiple patterns can be detected: for example aggregation or repulsion between entities according to the distance considered. These distance-based measures are now deemed to be very powerful and we can easily understand why many studies now employ them to evaluate spatial patterns (Arbia and Espa, 1996; Sweeney and Feser, 1998; Ó hUallacháin and Leslie, 2007; Bonneu, 2007; Arbia et al., 2008; Nakajima et al., 2012; Barlet et al., 2013; Koh and Riedel, 2014; Giuliani et al., 2014; Behrens and Bougna, 2015; Zhou and Clapp, 2015; Bade et al., 2015; Bocci and Rocco, 2016; Buzard et al., 2017). Alternative approaches exist. Billings and Johnson (2012) for instance developed a test to detect and localize concentration based on the local density of a chosen sector, compared to that of the whole economic activity. ${ }^{3}$ We will rather focus in this paper on the distance-based methods, which rely on the second-order property of the underlying point process, i.e. the excess or lack of neighbors, because they belong to a common, coherent framework (Marcon and Puech, 2017).

In what follows, we shall introduce a new distancebased method: the $m$ function. We will devote a great deal of attention to define it in order to respect a maximum number of the good criteria for measuring spatial concentration in economics (Combes and Overman, 2004; Bonneu and ThomasAgnan, 2015). In particular, the $m$ function satisfies Duranton and Overman's five important criteria (Duranton and Overman, 2005): (1) the results are comparable across industries, (2) it controls for the overall agglomeration of manufacturing, (3) it controls for industrial concentration in the sense of Ellison and Glaeser (1997), (4) the measure does not depend on an areal zoning (this is related to the MAUP issues) and lastly (5)

\footnotetext{
${ }^{3}$ Other developments may be cited as the one explained by Dubé and Brunelle (2014).
}

it gives an indication of the significance of the results. There are several ways to control for the overall agglomeration. Duranton and Overman's widely used $K_{d}$ function ignores it (this is why it is classified as an absolute measure) but its value is compared to a confidence interval of its possible values under a counterfactual null hypothesis: establishments are redistributed across the actual set of locations chosen. The $m$ function relies on the local share of employment (or whatever measure of size, including the number of establishments) of the sector under study to directly control for the distribution of the whole activity: it is a relative measure. As $K_{d}$, it considers neighbors at a given distance rather than up to it: both are density functions. No relative density function has yet been proposed to gauge the spatial concentration in continuous space, as Marcon and Puech (2012) pointed out.

We provide various simulated and real examples to show that the results provided by the $m$ function are complementary to those of $K_{d}$ : because the $m$ and the $K_{d}$ functions do not evaluate the notion of spatial concentration their results may be different. So we recommend that the nature of the spatial concentration analyzed should be studied carefully to avoid any erroneous conclusions. Moreover, one important feature of the $m$ function is that its values are interpretable. Relative measures of concentration are formally location quotients: the $m$ function can be interpreted as the location quotient of a sector of activity in the neighborhood of a reference sector. The possibility to interpret the results can be very appreciable for whom the objective is not only to detect spatial concentration or dispersion but also quantify it. This property opens the way for the development of locational choice models following Guimarães et al. (2009) where the strength of externalities or natural advantages can be directly linked to the value of the function. As a result, we believe the $m$ function is an appropriate statistical tool for detecting spatial structures in economics.

In the following sections, first of all, we explain precisely our motivation. Then, the $m$ function is presented and some simple illustrative examples are given. In the last section of the paper, we propose some comparisons with the $K_{d}$ function. We also provide a simple simulated example and an analysis of the spatial distribution of pharmacies in the Lyon area (France) to illustrate the advantages and limits of the use of the $m$ function in our field.

\section{Approach}

\subsection{Basic framework}

As we have already mentioned, distance-based methods are particularly attractive for economists because they provide a complete analysis of the location patterns of industries without a loss of information or modification of the raw dataset. The analysis is based on the spatial distribution of establishments (plants or shops). For each establishment of the sample analyzed, its characteristics are preserved: its exact location, its industry or any other individual characteristics (number of employees...). The idea of distance-based methods is to detect 
if there is an attraction or a repulsion between plants, based on an analysis on the bilateral distance between establishments. In order to understand the basic ideas of distance-based methods, let us consider for more clarity the case of the textile industry.

We first need to choose a null model for the density of textile plants everywhere in the area under investigation: the choice of the model will be detailed below. It defines the type of spatial concentration that is investigated: any departure from this null model is assumed to be due to interactions between plants. If there are locally more textile plants around textile plants than expected under the null model, distancebased methods will detect spatial concentration ("textile plants attract textile plants"). ${ }^{4}$ On the other hand, if there are fewer textile plants in the surroundings of the textile plants than expected, we identify a phenomenon of dispersion (in which case "textile plants repel textile plants"). ${ }^{5}$ Alternatively, if there is no relationship between the entities, independence is accepted ("textile plants are randomly and independently distributed").

The significance of the results is provided by the construction of the confidence interval of the null hypothesis. The latest is the null model of density in the absence of interactions between plants. For almost all empirical studies, this benchmark (also called counterfactual) is constructed by Monte-Carlo simulations to obtain a confidence interval of the results.

Finally note that the mathematical framework of distancebased methods is the one of point processes (Møller and Waagepetersen, 2004). Point processes are stochastic configurations. Loosely speaking they can be understood as random variables that generate realizations of spatial distributions of points (also known as point patterns). The first-order property of a point process is its intensity, i.e. the probability to observe a point at a given location, estimated by smoothing its observed number of points per unit area around this location. Its second-order property is the joint probability to find a couple of points at two locations, normalized by the probabilities to find each of them independently, i.e. without attraction or repulsion between pairs of points. Distance-based measures of spatial structures are designed to detect attraction or repulsion while controlling for intensity, according to an assumed null model.

\subsection{Motivation}

Two concepts require additional explanations for evaluating the spatial distribution of establishments: the definition of the surroundings of establishments and the nature of the spatial concentration (topographic, relative or absolute). Let us now examine these two important factors in depth.

Firstly, the notion of the surroundings of plants is central because it defines the type of function applied i.e. a cumula-

\footnotetext{
${ }^{4}$ The following terms: spatial concentration, concentration, agglomeration or aggregation are used as synonyms in this article.

${ }^{5}$ In the same way, dispersion or repulsion are synonyms.
}

tive or a density function. In practice, the evaluation of the neighboring plants is done for all distances, for example every 100 meters up to the median distance between all pairs of plants. The spatial distribution can be estimated up to a given distance or at a given distance. If the first option is chosen, it calls for a cumulative function. If the second option is selected, a density function is appropriate. The choice between one type of function and the other depends on the issue under study (Marcon and Puech, 2010).

The second clarification concerns the nature of the spatial concentration. In order to evaluate the spatial concentration of economic activities, it is necessary to choose a reference system in order to compare the observed distribution of activities (see Brülhart and Traeger, 2005, among others):

- The first possibility is to use a topographic reference. In this case, physical space is chosen as the benchmark. One possible example is the number of neighboring plants per unit of space (that is on a disk of radius $r$ for a cumulative function or on the ring at distance $r$ for a density function). Space may be homogeneous or not. The homogeneity of space implies a constant density all over the study area (in our previous case this means that all the plants in the distribution have the same probability of being located anywhere in the study area). Some authors (Duranton and Overman, 2005; Marcon and Puech, 2003) consider this hypothesis to be generally irrelevant in the field of spatial economics and that a non-homogeneous space framework is needed.

- The second possibility is a relative reference. In this case, another variable is taken as a benchmark. Any variable can be used except space (if it is, the concentration is topographic). For instance, if we evaluate the spatial distribution of textile plants, we can detect in the plant's near environment the deviations of this distribution of plants from another distribution. The benchmark plants can be all plants at the aggregate industrial level to fulfill Duranton and Overman's second criterion.

- The last possibility is to have no reference. In this case, an absolute measure is defined. For example, the number of neighboring textile plants located at a given distance from a textile plant. 
Table 1. Choice of the appropriate function to describe a point pattern structure

\begin{tabular}{|c|c|c|c|c|}
\hline Function choice & $\begin{array}{l}\text { Topographic, } \\
\text { homogeneous }\end{array}$ & $\begin{array}{l}\text { Topographic, } \\
\text { inhomogeneous }\end{array}$ & Absolute & Relative \\
\hline $\begin{array}{l}\text { Probability density } \\
\text { functions }\end{array}$ & $g$ & $g_{\text {inhom }}$ & $\begin{array}{c}K_{d} \\
K^{e m p}\end{array}$ & \\
\hline \multirow[t]{2}{*}{ Cumulative functions } & $K$ & $K_{\text {inhom }}$ & Cumulative of $K_{d}$ & $M$ \\
\hline & $K_{m m}$ & $D_{i}$ & Cumulative of $K^{e m p}$ & Case-control $K_{\text {inhom }}$ \\
\hline
\end{tabular}


The growing number of measures in continuous space recently prompted Marcon and Puech (2017) to provide a typology of such functions. A classification of statistical measures can be drawn up by considering the nature of the geographic concentration and the definition of the type of function. Table 1 gives an overview of all the distance-based measures that have been used to evaluate the spatial distribution of economic activities (Marcon and Puech, 2012):

- the $K$ function of Ripley $(1976,1977)$,

- the $g$ function of Ripley (1976, 1977),

- the $K_{m m}$ function introduced by Penttinen (2006) and Penttinen et al. (1992),

- the $D$ function of Diggle and Chetwynd (1991),

- the $K_{\text {inhom }}$ function of Baddeley et al. (2000),

- the $g_{\text {inhom }}$ function of Baddeley et al. (2000),

- the $K_{d}$ function and the $K^{e m p}$ function of Duranton and Overman (2005),

- the $M$ function of Marcon and Puech (2010),

- the (unnamed) cumulative function of $K_{d}$ proposed by Behrens and Bougna (2015).

One cell in the table is empty: no relative density function has yet been proposed for the field of spatial economics (the $K_{d}$ function does not control explicitly for the distribution of the economic activity). The present paper fills this gap. In the next section we shall complete Table 1 by proposing a new density function, named the $m$ function, which expresses relative spatial concentration.

The observed point pattern is always tested against the null hypothesis of independence between the location of points. If topographic concentration is studied, the intensity of the point process is estimated from the data (by simply dividing the number of points by the area if space is homogeneous, by kernel density estimation else for example) and an appropriate statistic (a topographic distance-based measure) is calculated from the data. The statistical significance of its departure from the expected value under the null hypothesis is tested by Monte-Carlo simulations of independent point patterns (i.e. Poisson point patterns) with the same intensity.

Relative measures assume that the intensity of the point process of interest (the sector under study) is proportional to its benchmark. Usually, the benchmark is the whole economic activity and the only available information is the observed location of all plants. Then, the null hypothesis is tested by randomly shuffling the marks of the points (types and weights together) among the observed points Duranton and Overman (2005).

Last, absolute measures do not have a predictable value under the null hypothesis. Instead, their observed value at each distance $r$ is compared to the quantiles of the same measure applied to point patterns simulated according to the null hypothesis. The $K_{d}$ function estimates the probability to find a neighbor of an arbitrary point, $r$ apart. This probability density is then compared to its quantiles in simulated point patterns obtained by shuffling the marks of the points.

Finally, it should be noted that the application of distancebased methods is not confined to spatial economics. They were first developed and applied in other disciplines. Much empirical research has thus been conducted in the fields of ecology (Law et al., 2009) and epidemiology (Waller, 2010), for example.

\section{Definition of the $m$ function}

\subsection{An intuitive presentation}

The idea of the $m$ function is as follows. Consider an area in which various plants belonging to several industrial sectors are located. The $m$ function is a relative measure that compares the proportion of plants of interest in the neighborhood of the reference plants to the proportion of neighbors of interest in the area as a whole. If plants are agglomerated, the proportion of neighbors of interest in the neighborhood of reference plants is greater than in the area as a whole. On the contrary, if plants are dispersed then the proportion of plants of interest in the neighborhood of the reference plants is lower than in the area as a whole. These proportions (ratios) are estimated from observed data. If the neighbors of interest belong to the same sector as the reference plants, the $m$ function helps to detect agglomeration phenomena. If the neighbors of interest do not belong to the same sector as the reference plants, the $m$ function identifies co-agglomeration.

\subsection{Mathematical definition}

Let us now turn to the mathematical definition of the $m$ function. Plants are defined as points. All points belong to a point pattern denoted by $\mathscr{X}$ which is a realization of a marked point process. In this framework, plants have a random location, a random sector of activity and a random size. Two subsets are considered: that of the reference points $\mathscr{R}$ (e.g. a given sector of activity) and that of the neighboring points of interest $\mathscr{N}$ (another sector, possibly the same).

The $m$ function of distance $r$ is the average ratio of the density of neighbors of interest (at distance $r$ from each reference point) to the density of all neighbors, normalized by the global proportion of points of interest.

Define $\lambda(x, r)$ the density of neighbors of an arbitrary point $x$ at distance $r$, i.e. the expected number of neighbors in a ring of width $d r$ and radius $r$ around point $x$ is $2 \pi r \lambda(x, r) d r . \lambda_{\mathscr{N}}(x, r)$ is the density of neighbors of interest. Note that neighbors can be plants or employees: in the first case, employees are represented by superimposed points at the same location or, equivalently, points representing plants are weighted by the number of employees. To fulfill Duranton and Overman's third criterion, neighbors must be weighted plants. 
$W_{\mathscr{N}}$ is the total weight of the neighboring points of interest and $W$ is the total weight of all the points.

The $m$ function is defined as an expectation:

$$
m(r)=\mathbb{E}\left(\frac{\lambda_{\mathscr{N}}(x, r) / \lambda(x, r)}{W_{\mathscr{N}} / W}\right)
$$

One natural estimator is:

$$
\hat{m}(r)=\frac{\sum_{x_{i} \in \mathscr{R}} \frac{\sum_{x_{j} \neq x_{i}, x_{j} \in \mathscr{N}} k\left(\left\|x_{i}-x_{j}\right\|, r\right) w\left(x_{j}\right)}{\sum_{x_{j} \neq x_{i}, x_{j} \in \mathscr{X}} k\left(\left\|x_{i}-x_{j}\right\|, r\right) w\left(x_{j}\right)}}{\sum_{x_{i} \in \mathscr{R}} \frac{W_{\mathscr{N}}-w\left(x_{i}\right)}{W-w\left(x_{i}\right)}}
$$

where $x_{i}$ denotes the reference points, and $x_{j}$ the neighbors. $w\left(x_{i}\right)$ is the weight of point $x_{i}$. If points represent industrial establishments or shops, the weight can be the number of employees working in those entities. $k(\cdot)$ is a kernel estimator whose sum can be used to estimate the number of neighbors of point $x_{i}$ at distance $r$. We have followed Duranton and Overman (2005) and used a Gaussian kernel with an optimal bandwidth proposed by Silverman (1986) as a rule of thumb. The kernel estimator considers the neighbors of the reference point and gives them a maximum weighting if they are exactly $r$ apart. Their weighting decreases according to the tail thickness of a Gaussian distribution with standard deviation $h$. The choice of the bandwidth $h$ is arbitrary but important (Illian et al., 2008). The wider it is, the smoother the estimator. In this article, we shall only analyze the spatial distribution of one sector, so we shall focus on the intratype (or univariate) $m$ function taking $\mathscr{R}=\mathscr{N}$. But we can extend the $m$ function for the analysis of inter-industrial spatial distributions (co-agglomeration): the intertype (or bivariate) function can be defined in the same way, choosing different point types as the reference and neighbors. ${ }^{6}$

The equation of the $m$ function reads as follows. The numerator is the sum of the local ratios i.e. the relative weight of the neighbors of interest at distance $r$ from all the reference points. This is averaged over all the reference points (actually, it is simply summed because the number of reference points is simplified with the denominator). The denominator is the same ratio over the whole data set, i.e. the global ratio. It is obtained when $r$ is great enough for all points to be neighbors. It is not just $W_{\mathscr{N}} / W$ because the reference points are never counted as neighbors: their weight must be subtracted from the total weights. The denominator is slightly different in the intertype function: $\sum_{x_{i} \in \mathscr{R}} \frac{W_{\mathscr{N}}}{W-w\left(x_{i}\right)}$ because reference points are not counted in $W_{\mathscr{N}} / W$.

The benchmark value of the $m$ function is 1 for any distance $r$. This value is obtained when the types of points (and the size of points) are uniformly distributed random among all

\footnotetext{
${ }^{6}$ To give an example, if the aim is to evaluate the spatial distribution of the textile industry, the analysis of the distribution of textile plants around textile plants is relevant. In that case of intra-industrial analysis, the intratype function should be used. If the focus is now on the co-agglomeration of the textile and clothing sectors, the intertype functions will deal with the distribution of textile plants around clothing plants or the distribution of clothing plants around textile plants.
}

the considered points. $m$ values greater than 1 indicate the relative spatial concentration of points while $m$ values lower than 1 express relative dispersion. $m$ values can be interpreted. For example, if the $m$ function is 1.5 , at distance $r$, the proportion of the neighbor points of interest at this distance is $50 \%$ higher than in the area as a whole. As one can note, the interpretation of $m$ values is possible and is very easy. This property can be very appreciable if the aim is not only to detect spatial concentration or dispersion but to evaluate them. This can be useful for example for the comparison of the importance of the agglomeration across industries, for the explanation of the determinants of agglomeration (in the spirit of the study of Rosenthal and Strange (2001) with the Ellison and Glaeser index for example) etc.

The significance of the estimates of $m$ is given by the confidence interval of the null hypothesis (Monte-Carlo simulations). This technique is widely employed in the case of distance-based methods. In practice, random patterns of points are generated by permuting the marks (type and weighting pairs) of the actual points on the actual spatial positions of points (coordinates). We generate only a global confidence interval, following Duranton and Overman (2005).

\subsection{Properties}

The $m$ function fulfills all of Duranton and Overman's criteria mentioned in the introduction Duranton and Overman (2005): (i) it compares the geographic concentration results across industries, (ii) it controls for industrial concentration (indirectly, comparing its values to the confidence envelope of the appropriate null hypothesis), (iii) it controls for the overall aggregation patterns of industries, (iv) it enables the significance of the results to be tested (using the confidence interval) and, (v) data are analyzed without loss of information or modification of the raw dataset. Only a few continuousspace based methods respect all of these criteria (Marcon and Puech, 2017).

In continuous space, the definition of $m$ is similar to that of the cumulative $M$ function (Marcon and Puech, 2010) except that the local ratio is defined at distance $r$ and not up to it. In contrast with the topographic functions $g$ and $K$, the cumulative function is not the integral of the density function over $r$ (Ripley, 1977) because relative functions are not derived from a measure of space. The $m$ function can be interpreted as an extension to continuous space of the location quotient (Florence, 1972). It is not a smoothed Ellison and Glaeser's index: the latter relies on the squared difference between the local share of the sector of interest and that of the whole activity, not on their ratio.

\subsection{Inhomogeneity or interactions}

To conclude this section, we would like to pay a particular attention on the distinction between inhomogeneity and attraction or repulsion that are responsible for apparent spatial structure of real data.

Aggregates of plants on a map may be obtained according to completely different models: let's consider clothing shops 
in a given city and coal mines located in a given area. In the first case, the pattern of clothing shops should be compared to that of all (non-food) retail shops of the city. Clothing shops settle close to each other to increase their attractiveness for consumers and both $m$ and $K_{d}$ are able to detect aggregation properly.

Agglomeration may also be caused by inhomogeneity. In the second case, the benchmark pattern may be that of all industrial plants but the pattern of mines is actually driven by resources: they do not aggregate because of mutual attraction but because of the presence of coal, i.e. inhomogeneity of the underlying point process. There is no way to disentangle inhomogeneity and attraction from a single point pattern: several realizations of the process would be necessary to know whether the clusters always occur at the same place (coal resources) or not (clothing shops may aggregate anywhere).

Distance-based functions rely on the assumption that the excess or lack of neighbors is due to attraction or repulsion between points (Marcon and Puech, 2012). In other words, using a distance-based function to detect aggregation or repulsion implies assuming that the intensity of the point process that generated the observed point pattern is correctly controlled for. More specifically, relative functions such as $m$ assume that the sector under investigation has an intensity proportional to that of the whole economic activity it is compared to. We may call this property relative homogeneity.

\section{Application}

We shall now provide simple examples for three simulated cases. In every example we have considered a 1-by-1 window and a maximum distance for the $m$ function equal to one-third of the diagonal of the window $(\approx 0.471)$. We have systematically used 512 regular intervals to calculate the $m$ function. In all simulated examples, we generate two point patterns: the cases and the controls. ${ }^{7}$ The cases are the points of interest. The controls are the points that constitute the benchmark. For simplicity, all points have a weighting of 1 . A global confidence interval (CI) at the $1 \%$ risk level was generated after 10,000 simulations. All simulations are made with the help of the R software (R Development Core Team, 2018). The package spatstat (Baddeley and Turner, 2005) was used to simulate the point processes and the dbmss package (Marcon et al., 2015) was employed to compute the $m$ function.

In these examples, the cases represent plants of the sector under investigation and the controls the plants of other sectors. For simplicity, both are drawn in homogeneous processes, so that aggregates are visible on maps and the main features of the function appear clearly. Relative functions are designed to deal with non constant intensity of the controls, introduced in the next section where more complex situations will be studied.

\footnotetext{
7 The vocabulary "cases" and "controls" is well established in the literature of point processes (Diggle, 1983; Arbia et al., 2012) but some authors as Billings and Johnson (2012) prefer employing respectively "samples" and "counterfactuals".
}

\subsection{Independent cases}

Figure 1a shows two spatial point patterns: one for the cases (diamonds) and the other for the controls (crosses). The cases and controls were simulated under the hypothesis of complete spatial randomness (CSR), under which points are distributed randomly and independently from each other. To achieve this, we generated two patterns from a homogeneous Poisson process with a parameter respectively equal to 25 and 100 . The parameter of the Poisson process is the expectation of the number of points per unit area. This parameter is called the intensity of the process and it is constant in a homogeneous framework $^{8}$. In figure 1a, 29 points were simulated for the cases and 103 for the controls.

Cases are distributed with an intensity proportional to that of the control, thus analyzing them with a relative function is appropriate, and independently from each other, thus we expect $m(r)$ equal to 1 at all distances.

Figure 1b depicts the $m$ function results for this case. No significant departure from the null hypothesis is observed: $m$ fluctuates for all distance ranges but stays inside the confidence interval of the null hypothesis. As expected for random distributions of cases and controls, figure $1 \mathrm{~b}$ provides no evidence of any concentration or dispersion between cases. Two additional minor comments should be made. First, the global confidence interval is quite large at small radii: there is a small number of neighbors at very small radii. Second, the $m$ function is not defined for very small distances: this indicates that cases are separated by gaps of more than 0.01 .

\subsection{Aggregated cases}

Figure 2a shows a multiple pattern: an aggregate pattern of cases (diamonds) and a completely random distribution of controls (crosses). For the clusters of cases, we generated simulations from a Matérn process with the following parameters: 2 for the intensity of the Poisson process that generates cluster centers, 0.05 for the radius of clusters and 50 for the average number of points per cluster. Controls were simulated from a homogeneous Poisson process with a density of 100. 185 points were plotted on figure $2 \mathrm{a}$ : 83 cases shared between two clusters and 102 controls were randomly distributed over the entire domain. Figure $2 \mathrm{~b}$ shows the results for the $m$ function.

On figure $2 b$, two significant concentration zones appear. They occur at distances for which the relative local density of cases is the greatest. The first distance at which a maximum is observed corresponds approximately to the radius of the clusters (around 0.05). The second zone identifies the distance between clusters (approximately 0.35) and has a lower value. The local relative density of cases over controls is greater for the first peak because the presence of controls in the cluster is possible but rare.

\footnotetext{
${ }^{8}$ The Poisson process is commonly used for simulating CSR patterns. As Diggle (1983) wrote the Poisson process "is the cornerstone on which the theory of spatial point processes is built. It represents the simplest possible stochastic mechanism for the generation of spatial point patterns, and in applications is used as an idealized standard of complete spatial randomness $(\ldots) "(\mathrm{p} .50)$.
} 


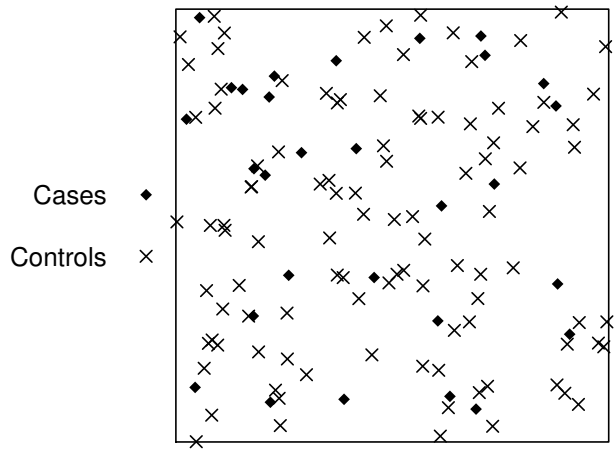

(a) Completely random patterns for cases and controls

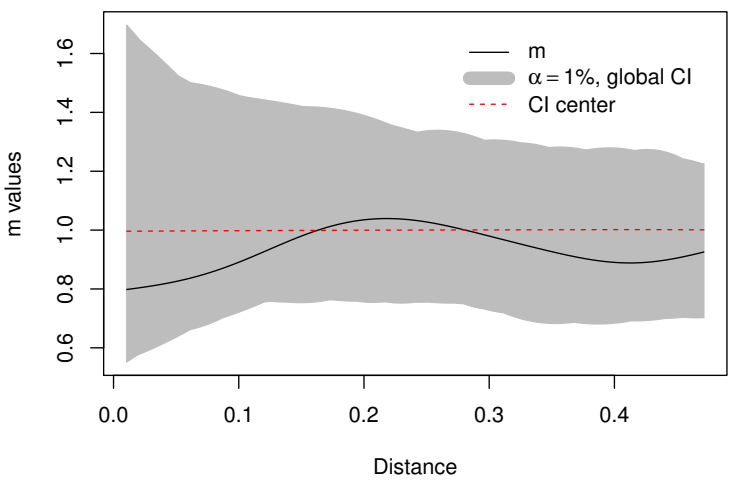

(b) $m$ function results

Figure 1. Random point pattern

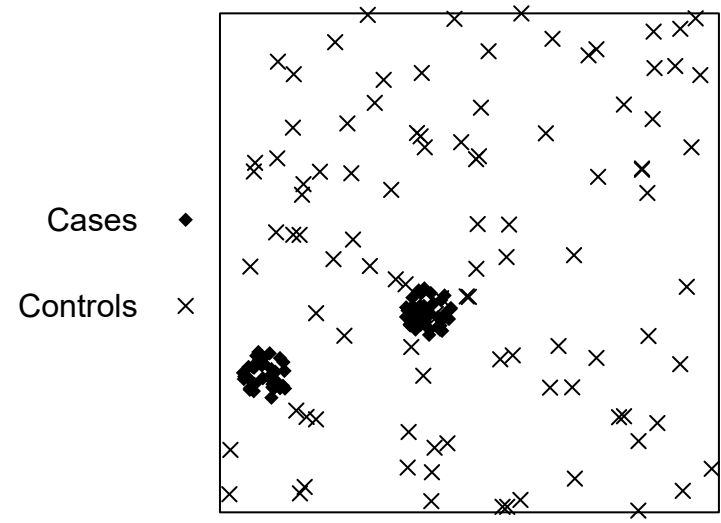

(a) Aggregate pattern of cases, complete spatial randomness for controls

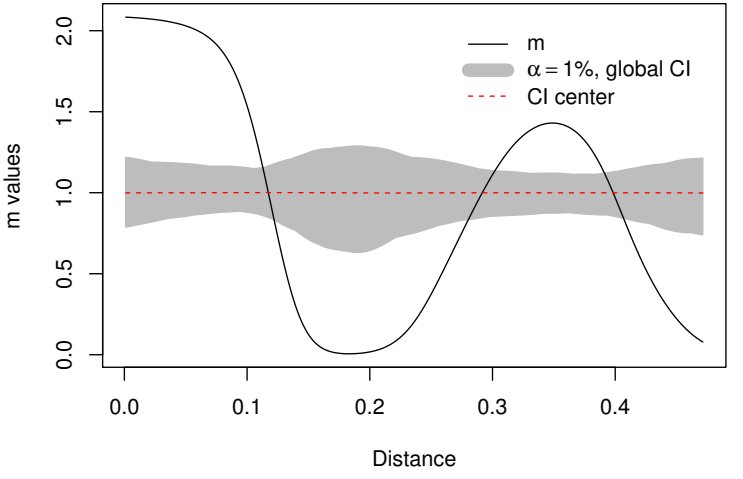

(b) $m$ function results

Figure 2. Aggregate point pattern 
Three additional comments on this case have to be made.

First, by construction there are no cases between the aggregates. For these distances, the maximum dispersion is detected: between the clusters the $m$ function attains its lowest possible value (zero). The rapid decrease in the gradient of $m$ is a feature of density functions. In contrast to cumulative functions, the values are very sensitive and large ranges of results may be observed over small intervals of distance.

Second, one can observe that the $m$ plot takes its highest values in the case of small distances (first concentration peak) and then decreases. The explanation for this is simple. In the first radii, the local relative density is the greatest because the maximum number of cases is observed around these distances. Around a distance of 0.2 , the $m$ function detects the first cases located at the periphery of the (other) cluster: as a result, the $m$ function raises. Then the local relative density continues to increase rapidly because of the large number of cases inside the cluster, but the presence of controls in the ring lowers the value of the $m$ plot at a distance of 0.35 . After this distance, the $m$ plot decreases rapidly because of the absence of cases and goes to its lowest possible value (zero).

\subsection{Regular cases}

Figure 3a shows another multiple pattern. A regular pattern of cases (diamonds) is clearly visible. 100 cases are positioned on a square grid with cells measuring $0.1 \times 0.1$. The completely random distribution of controls (crosses) is a realization of a homogeneous Poisson process whose parameter is equal to 200. Figure 3a shows 209 controls. The $m$ function estimates are given in figure $3 \mathrm{~b}$.

Up to the size of the square grid $(0.1)$, the cases have no case neighbor: for small distances there is a significant amount of dispersion. Then, a large number of peaks can be observed but results are not significant. The reason is simple. In this example as we previously said we retained the optimal bandwidth as described by Silverman (1986). A thinner bandwidth would have shown significant positive and negative peaks. The choice of the bandwidth is important, unfortunately "in general, however, no simple recipe for the choice of the bandwidth exists" (Illian et al., 2008, p.115). Let us take half of the previous bandwidth to better explain the spatial structure under study. Results of the $m$ function are given in figure $3 c$. Up to the size of the square grid $(0.1)$, $m$ plots are the same whatever the definition of the bandwidth. At a distance equal to the size of the grid, the cases have four neighbors: a significant positive peak is observed in figure $3 \mathrm{c}$, indicating the spatial concentration of cases at this distance. At this distance, a positive peak is also detected in figure $3 \mathrm{~b}$ but the $m$ plot stays within the confidence interval. Due to smoothing, significant values of $m$ can also be observed just below the grid size (0.1). The $m$ plot then plummets when the radius increases: no cases are located in the close environment of cases, the $m$ value returns rapidly to below the confidence interval, indicating dispersion. On figure $3 c$, the irregularity in the gradient of $m$ between a distance of 0.10 and 0.15 is interesting. At a distance equal to the diagonal of the grid (around 0.141) new neighbors are present. The irregularity in the gradient of $m$ shows the existence of these new neighboring points. However, there is no positive peak because the smoothing we applied was too strong, but weaker smoothing would have generated positive $m$ values. The original bandwidth appears too large in that case: the irregularity in the gradient of $m$ is not visible between a distance of 0.10 and 0.15 in figure $3 \mathrm{~b}$. Note at larger distances, the observed positive peaks appear at around twice the grid size $(0.2)$, three times the grid size (0.3) etc. Between these peaks, $m$ indicates the absence of neighboring cases: depending on the distance, significant dispersion may (as in the case for a distance of 0.25 ) or may not (as in the case for a distance around 0.35 ) be observed.

\section{Discussion}

This section provides some comparisons with the most used density function in spatial economics, Duranton and Overman's $K_{d}$ function (Duranton and Overman, 2005). Keeping the notations previously used in equation 1 and using $n$ to denote the total number of points, the $K_{d}$ function is defined by:

$$
\hat{K}_{d}(r)=\frac{1}{n(n-1)} \sum_{x_{i} \in \mathscr{R}} \sum_{x_{j} \neq x_{i}, x_{j} \in \mathscr{N}} k\left(\left\|x_{i}-x_{j}\right\|, r\right)
$$

The weighted version of the $K_{d}$ function, called the $K^{e m p}$ function (Duranton and Overman, 2005), is given by:

$$
\hat{K}^{e m p}(r)=\frac{\sum_{x_{i} \in \mathscr{R}} \sum_{x_{j} \neq x_{i}, x_{j} \in \mathscr{N}} w\left(x_{i}\right) w\left(x_{j}\right) k\left(\left\|x_{i}-x_{j}\right\|, r\right)}{\sum_{x_{i} \in \mathscr{R}} \sum_{x_{j} \neq x_{i}, x_{j} \in \mathscr{N}} w\left(x_{i}\right) w\left(x_{j}\right)}
$$

The $K_{d}$ function is very popular in spatial economics (Chain et al. (2019) or see Marcon and Puech (2017) for a review). It is therefore interesting to compare their properties in order to understand the main differences between the two statistical measures. In what follows, we have used the R package dbmss to estimate the $K_{d}$ and $m$ functions. As suggested by Duranton and Overman (2005), we use the reflection technique ${ }^{9}$ to estimate density close to the lowest distance for the $K_{d}$ function. As a result, $K_{d}$ plots start systematically for $r=0$.

\subsection{Comparisons of $K_{d}$ and $m$ results on the three previous simulated cases}

The $K_{d}$ function was estimated for the three simulated cases considered above. $K_{d}$ plots are shown on figure $4 \mathrm{a}$ for the

\footnotetext{
${ }^{9}$ In a few words, densities are underestimated around the limits of the interval. This is due to the fact that outside the interval, densities are not equal to zero as they should be. This border effect problem is known (Silverman, 1986) and can be easily corrected in practice by using for example the GoFKernel package (Pavia, 2015) for the R software. The idea is to use the reflection at the borders to correct the underestimated densities inside the interval but around the limits of the interval.
} 


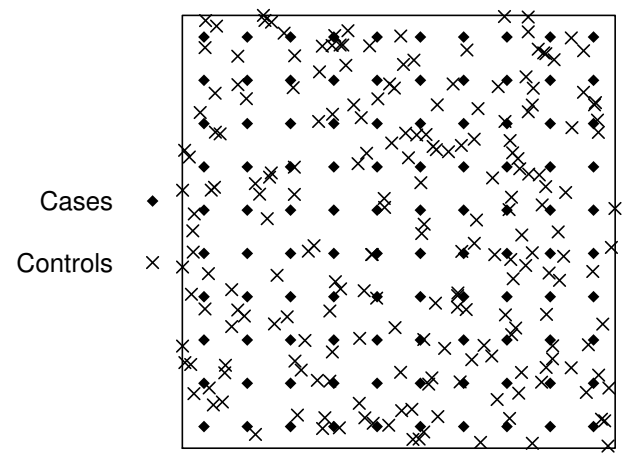

(a) Regular pattern of cases, complete spatial randomness for controls

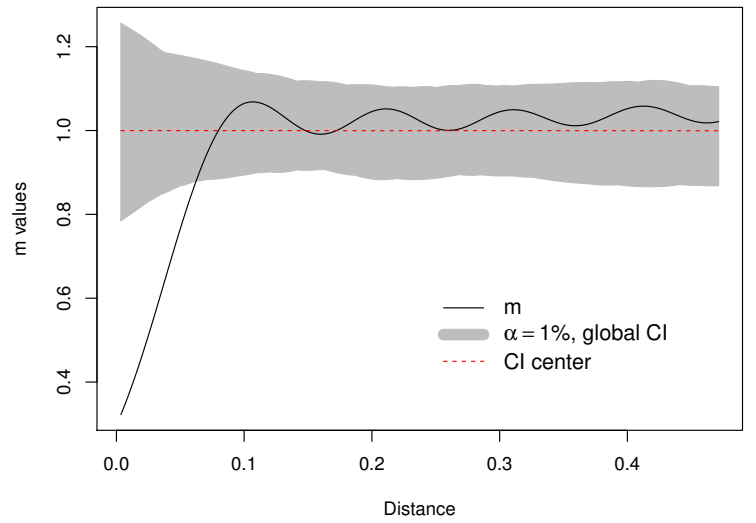

(b) $m$ function results, original bandwidth of Silverman (1986)

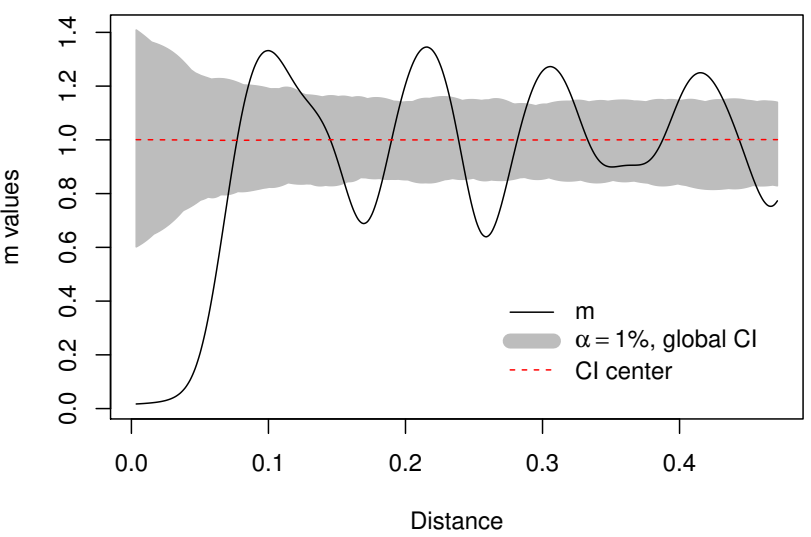

(c) $m$ function results, half of the original bandwidth of Silverman (1986)

Figure 3. Regular point pattern 


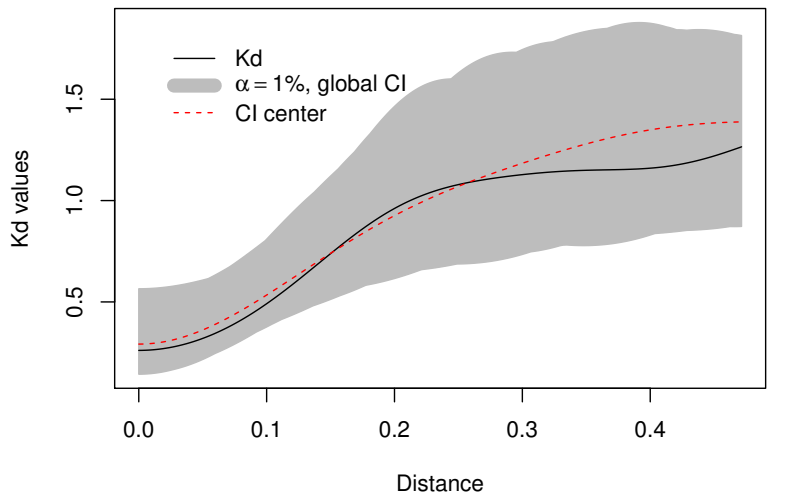

(a) $K_{d}$ results for completely random patterns of cases and controls (map on figure 1a)

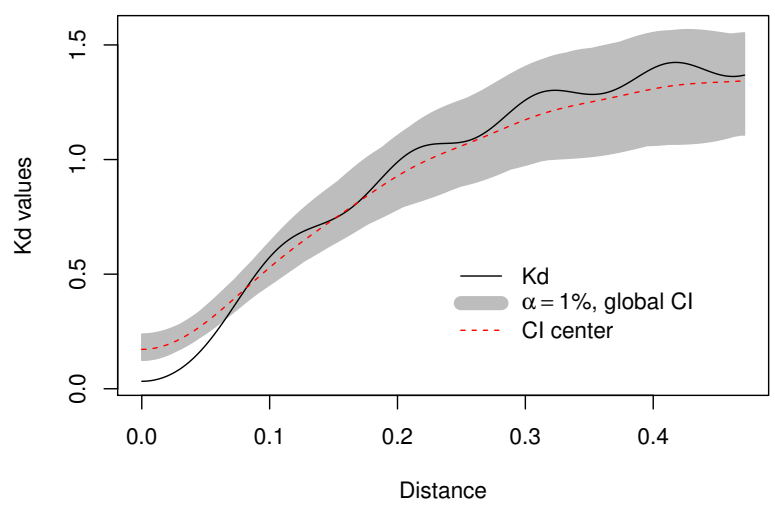

(c) $K_{d}$ results for regular pattern of cases (map on figure 3a) with the original Duranton and Overman's smoothing

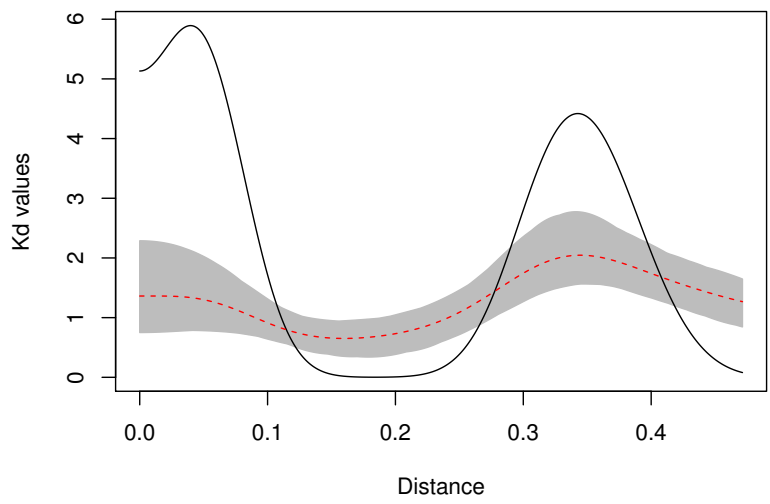

(b) $K_{d}$ results for aggregate pattern of cases (map on figure 2a)

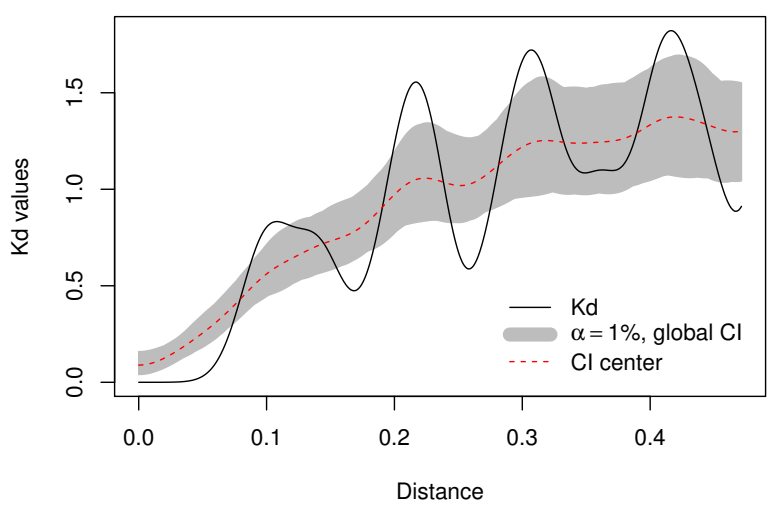

(d) $K_{d}$ results for regular pattern of cases (map on figure 3a) with a more detailed smoothing

Figure 4. $K_{d}$ results for the three simulated cases 
random pattern of cases, on figure $4 \mathrm{~b}$ for the aggregate pattern and on figures $4 \mathrm{c}$ and $4 \mathrm{~d}$ for the regular pattern. For all the cases, we have used the same maximum distance, i.e. onethird of the diagonal of the window $(\approx 0.471)$.

For the random patterns of cases and controls (figure $4 \mathrm{a}$ ), $m$ and $K_{d}$ give identical results. No significant level of dispersion or concentration was detected. The value of $m$ is 1 for all distances, subject only to stochastic fluctuations. Relative distance-based measures do not suffer edge-effects: points close to the domain borders have less neighbors but this issue cancels out when the ratio of their numbers is calculated. In contrast, $K_{d}$ increases with distance for geometrical reasons. $K_{d}$ evaluates the probability of finding a case neighbor at a given distance, i.e. on the circle of radius $r$ around each point of interest: it first increases linearly with respect to $r$, then increases less because of edge effects (parts of the circles lay outside the domain when $r$ is large enough) and finally drops to 0 when $r$ gets larger than the diameter of the domain (not shown on the figure). Finally note a very minor difference in the results provided by $K_{d}$ and $m$ : the $K_{d}$ plot is defined for $r=0$ contrarily to the $m$ plot (figure $1 \mathrm{~b}$ ). This is due to the reflection method used for the $K_{d}$ function as we previously explained. No case-neighbor is located at a distance less than 0.01 thus $m$ is not defined.

For the aggregate pattern (figure 4b), like the $m$ function, $K_{d}$ detects the first peak of concentration occurring at a distance of approximately 0.05 . The main difference relates to the shape of the concentration peak. At very small distances the $K_{d}$ values increase up to a distance of 0.05 which corresponds to the radius of the cluster. After this, the value of $K_{d}$ starts to fall. The increase in $K_{d}$ contrasts with the shape of the $m$ function at short distances. The explanation is geometric again: at short distances this probability increases proportionally to the perimeter of the circle around reference points until $r$ is too large and the circle partly leaves the cluster. Then, it progressively decreases.

Let us now turn to the regular simulated example. With the same original smoothing of Silverman (1986) also chosen by Duranton and Overman (2005), the results for this spatial pattern with the $m$ (figure $3 \mathrm{~b}$ ) or the $K_{d}$ (figure $4 \mathrm{c}$ ) function are totally in accordance. If we modify the smoothing by choosing a narrower bandwidth, that is half of the original bandwidth of Silverman (1986), the results for the $K_{d}$ function are given in figure $4 \mathrm{~d}$ and should be compared with the $m$ results given in figure $3 \mathrm{c}$. As one would expect, there are a large number of positive and negative significant peaks of the $K_{d}$ plot in comparison to weaker smoothing of the results. The $m$ results and the $K_{d}$ results are, again in that example, totally in accordance.

\subsection{Comparisons between the results from the $K_{d}$ and $m$ functions on a more complex example}

In the three above simulated cases, there is not a great deal of difference between the results with the $K_{d}$ and $m$ functions. However, this is not always the case. In the real world, the distribution of activities is more complex. In this sub-section, we shall draw attention to the type of concentration that the $m$ function can identify. To make things clearer, if we return to table 1 we can see that the $m$ function evaluates the relative concentration while $K_{d}$ appraises the absolute concentration. This distinction may be crucial for a comprehensive understanding of spatial structures.

\subsubsection{Divergence in the $K_{d}$ and $m$ results on a simulated case}

Consider the following simulated example. A city is delimited by a 1-by-1 window. For the sake of simplicity "cases" and "controls" are the only two types of shops located in the city. Figure 5 shows the distribution of cases (diamonds) and controls (crosses). A multiple pattern is observed: a completely random distribution of controls (crosses) and cases (diamonds) and also an aggregate distribution of controls (crosses). More technically, for the cluster of controls we generated simulations from a Matern process with the following parameters: 1 for the density of the Poisson process that generates cluster centers, 0.1 for the radius of clusters and 75 for the average number of points per cluster. Controls were also simulated from a homogeneous Poisson process with a density of 50. Cases were simulated from a homogeneous Poisson process with a density of 25.134 points were plotted on figure 5 . The cluster is composed of 66 controls, 42 controls are randomly distributed on the area and 26 cases are randomly distributed over the entire domain.

Figures $6 \mathrm{a}$ and $6 \mathrm{~b}$ show the results for the $K_{d}$ function and the $m$ function. In line with our previous examples, all points have a weighting of 1 . The maximum distance for the $m$ function equals one-third of the diagonal of the window $(\approx 0.471)$. A global confidence interval $(\mathrm{CI})$ at the $1 \%$ risk level was generated after 10,000 simulations. All simulations were conducted with the help of the dbmss package for computing the $K_{d}$ and $m$ functions. The divergence in the results between $K_{d}$ and $m$ plots show all the importance of the definition of the nature of the spatial concentration studied.

As we underlined, the $m$ function detects the relative spatial concentration. Cases are more regularly distributed than the controls in the example. As a consequence, around the cases the presence of cases is relatively more important than the one of controls: relative concentration is detected by the $m$ function up to a distance approximately equal to 0.23 (figure $6 \mathrm{~b}$ ). Now, consider the $K_{d}$ results (figure 6a): dispersion of cases is detected. By construction of the example, cases do not take place in the clusters. Under the null hypothesis of random location of cases among all observed points, some cases will be located in the cluster: the probability to find neighbors at short distances is thus higher than in the real data set; in other words, the points of the dataset are less concentrated than under the null hypothesis. The concentration characterized by $K_{d}$ is called absolute because it just counts neighbors without comparing their number to a benchmark. In the dataset, cases are located in low-density areas so they are far from each other: a dispersion of cases is detected by 


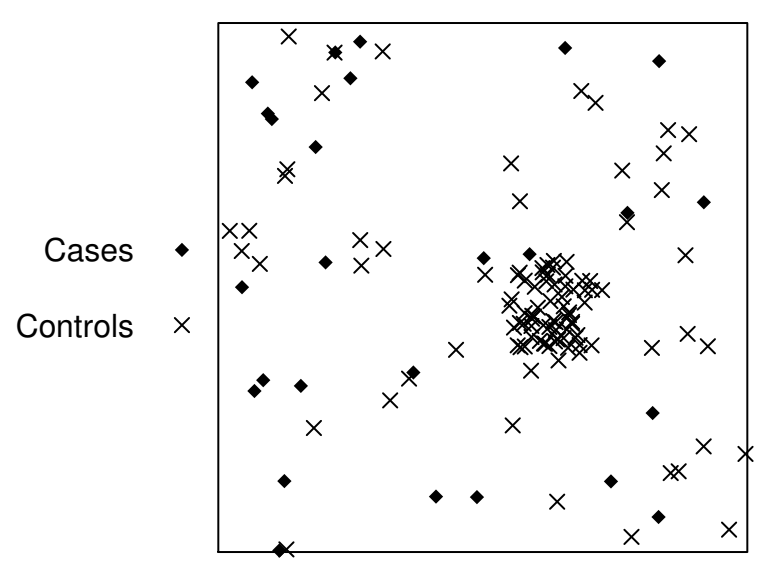

Figure 5. A more complex simulated case

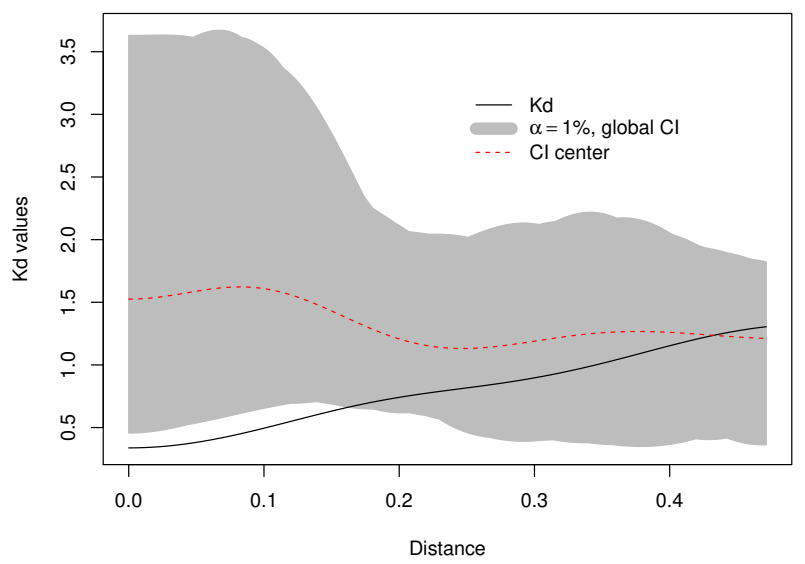

(a) $K_{d}$ function results

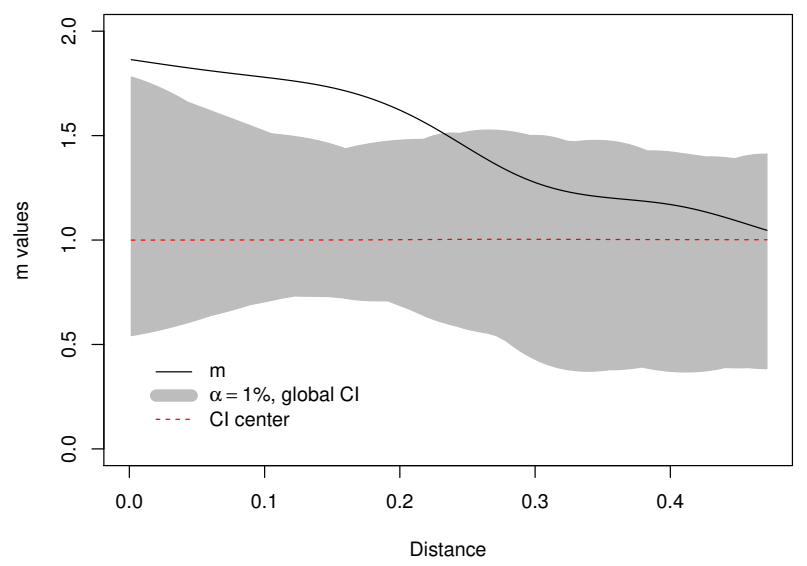

(b) $m$ function results

Figure 6. $K_{d}$ and $m$ results for a more complex simulated case 
$K_{d}$. However, they are relatively abundant and close to each other (in comparison with controls), cases are agglomerated in relative terms, as detected by the $m$ function.

\subsubsection{Confirmation of the previous results for a retail sec- tor in the city of Lyon (France)}

To give a concrete example of the previous simulated case, we shall consider the spatial distribution of the non-food retail stores in the Lyon area (France). We have exploited a database provided by the Chamber of Commerce and Industry of Lyon. This contains the exact geographic position of 3,124 non-food stores in April 2012. The different types of store have been classified into 26 sectors from $47.30 \mathrm{Z}$ to $47.79 \mathrm{Z}$ of the French NAF rev. 2 classification of activities. We shall focus on "dispensing chemists in specialized stores" (47.73Z), of which there are 156 in the Lyon area. We shall refer to these stores as pharmacies in what follows.

First of all, a comparison of the density of all non-food stores in Lyon (figure 7a) and the spatial distribution of the pharmacies over the same area (green crosses on figure $7 \mathrm{~b}$ ) is worthwhile. We can see that many of the city's non-food stores are located in central Lyon and the left bank of Rhône river (to the east of central Lyon, figure 7a). However, pharmacies are undoubtedly more regularly distributed than non-food stores as a whole. One can easily observe the presence of these activities over the entire Lyon area (black points on figure $7 b$ ).

The impacts on the results for $m$ and $K_{d}$ will be of interest. These results are given on figures $8 \mathrm{a}$ for $K_{d}$ and $8 \mathrm{~b}$ for $m$ (distances are reported in meters on the horizontal axis). Their respective global confidence intervals $(\mathrm{CI})$ were computed at the $1 \%$ risk level after 10,000 simulations. All the pharmacies were assigned a weight of 1 and the maximum distance analyzed was around 2,500 meters. The spatial structures detected by $K_{d}$ and $m$ differ. Up to a distance of approximately 2 kilometers, the plot of $K_{d}$ indicates that pharmacies are dispersed while that of $m$ indicates a degree of spatial concentration up to 1.5 kilometers. The peak of spatial concentration appears around 750 meters and is nearly equal to 1.5 which means that the density of pharmacies at this radius is $50 \%$ higher than in the Lyon area as a whole. How can we explain this difference in the results of $K_{d}$ and $m$ ? As we underlined, pharmacies are more regularly distributed than non-food retail activities as a whole. As a result, pharmacies are more concentrated under the null hypothesis than in the real distribution: a dispersion of pharmacies is detected by $K_{d}$. Moreover, even though there are pharmacies in high density business areas (central Lyon, the left bank of the River Rhône... ), they are over-represented in low-density business areas, relatively to other shops. When we simulate distributions, pharmacies are located in areas where the number of non-retail stores is greater so the relative concentration of these stores will be lower under the null hypothesis. As a result, the observed $m$ plot is over the confidence interval of the null hypothesis and indicates a relative spatial concentration of pharmacies between approximately 250 meters and 1,500 meters.

\subsubsection{Synthesis}

The purpose of both $K_{d}$ and $m$ functions is to compare the distribution of a specific sector of activity to that of the economic activity a whole, to control for its obvious spatial heterogeneity. Explicitation of the null hypotheses allows understanding why their results may detect spatial concentration or dispersion.

The $m$ function estimates the relative concentration (or dispersion, omitted in the rest of the discussion for readability) of the sector of interest, i.e. it compares the average proportion of neighbors of this sector around all plants or shops of this sector to its global proportion. Significant values of $m(r)$ detect relative concentration. The $K_{d}$ function compares the probability to find a neighbor of this sector around the same points to the probability that would be under the null hypothesis of random location of plants. Significant departures of $K_{d}$ from the null hypothesis show that the topographic concentration of the sector is higher than that of the the benchmark. It focuses on topographic, not relative concentration.

This comparison of the $m$ and $K_{d}$ functions emphasizes that the nature of the spatial concentration should be studied with care to avoid any misinterpretation.

\section{Conclusion}

In this article, we introduced a new distance-based function called $m$. The $m$ function is the first relative density function to be proposed in the field of spatial economics. It respects all of the good criteria of Duranton and Overman (2005) for evaluating the spatial distribution of economic activities. So, $m$ will certainly be useful for economists for detecting the relative spatial concentration or dispersion of activities. Density functions detect local patterns more precisely than cumulative functions so $m$ can be preferred in that case to relative cumulative distance-based measures. Moreover, we showed on simulated and real data that $m$ and the leading density function $K_{d}$ of Duranton and Overman (2005) may be used conjointly for having a comprehensive approach of the distribution of activities. The main reason is that $K_{d}$ compares the topographic concentration of a sector to that of the whole activity while $m$ actually evaluates the relative concentration of the sector. At the end of the article, the analysis of the distribution of the pharmacies in Lyon provides a good example of the complementary of the results of $K_{d}$ and $m$. 


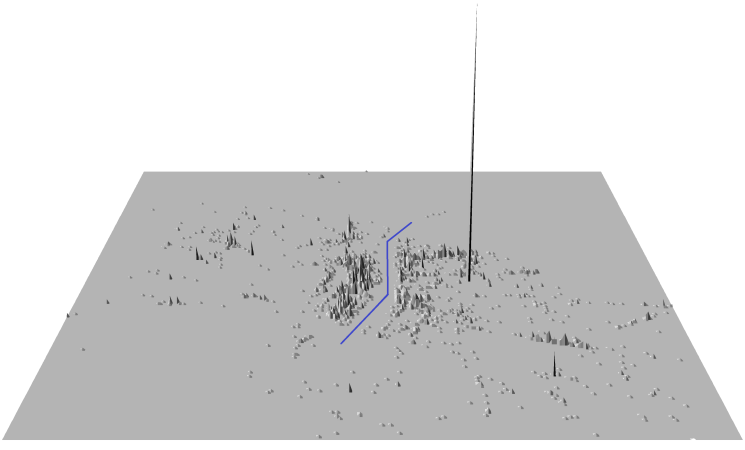

(a) Density of the non-food retail stores. The blue line represents the position of the Rhône River.

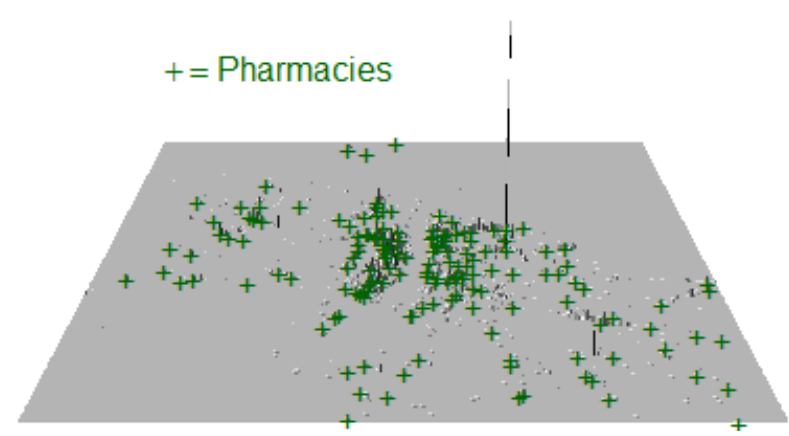

(b) Spatial distribution of pharmacies in Lyon

Figure 7. Non-food retail stores in the area of Lyon (France)

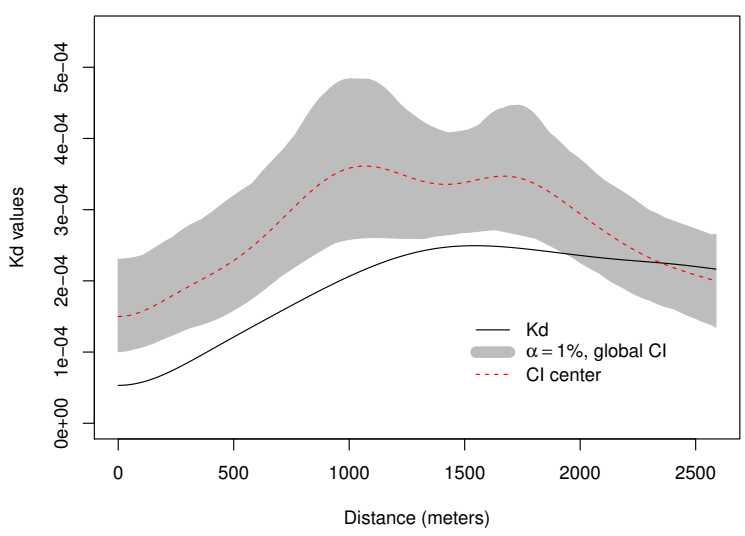

(a) $K_{d}$ results for pharmacies in Lyon

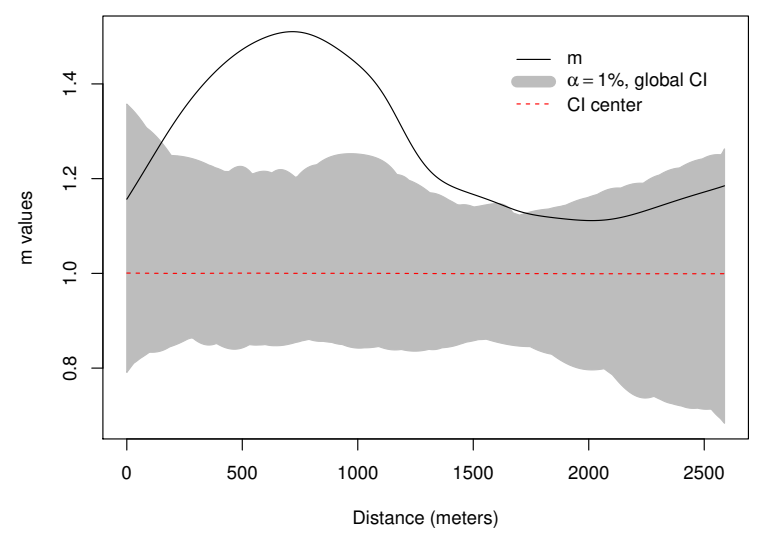

(b) $m$ results for pharmacies in Lyon

Figure 8. Spatial structure of the pharmacies 


\section{Acknowledgments}

Thanks to the Guest editors of this special issue and to the two anonymous referees for their comments. We are grateful to the Lyon Chamber of Commerce and Industry and Christophe Baume for providing us with data on stores. We also thank those who took part in the International Workshop on Spatial Econometrics and Statistics (Toulon, France), the Workshop on spatial statistics: Measurement of spatial concentration and its applications (Sceaux, France) and the Annual North American Meetings of the Regional Science Association International (Washington D.C., USA) for their valuable comments and discussions.

This work was supported by the AAP Attractivite 2014 (Université de Paris-Sud), the Paris-Saclay Center for Data Science funded by the IDEX Paris-Saclay, ANR-11-IDEX0003-02, and an "Investissement d'Avenir" grant managed by Agence Nationale de la Recherche (CEBA, ref. ANR-10LABX-25-01).

\section{References}

Alfaro L, Chen MX (2014) The global agglomeration of multinational firms. Journal of International Economics 94(2):263-276

Arbia G (1989) Spatial Data Configuration in Statistical Analysis of Regional Economic and Related Problems. Kluwer, Dordrecht

Arbia G (2001) The Role of Spatial Effects in the Empirical Analysis of Regional Concentration. Journal of Geographical Systems 3(3):271-281

Arbia G (2016) Spatial Econometrics: A Broad View. Foundations and Trends $\AA$ in Econometrics 8(3-4):145-265

Arbia G, Espa G (1996) Statistica economica territoriale. Cedam, Padua

Arbia G, Espa G, Quah D (2008) A class of spatial econometric methods in the empirical analysis of clusters of firms in the space. Empirical Economics 34(1):81-103

Arbia G, Espa G, Giuliani D, Mazzitelli A (2012) Clusters of firms in an inhomogeneous space: The high-tech industries in Milan. Economic Modelling 29(1):3-11

Baddeley AJ, Turner R (2005) Spatstat: an R package for analyzing spatial point patterns. Journal of Statistical Software 12(6): $1-42$

Baddeley AJ, Møller J, Waagepetersen RP (2000) Non- and semi-parametric estimation of interaction in inhomogeneous point patterns. Statistica Neerlandica 54(3):329-350

Bade FJ, Bode E, Cutrini E (2015) Spatial fragmentation of industries by functions. The Annals of Regional Science 54(1):215-250
Barlet M, Briant A, Crusson L (2013) Location patterns of service industries in France: A distance-based approach. Regional Science and Urban Economics 43(2):338-351

Behrens K, Bougna T (2015) An Anatomy of the Geographical Concentration of Canadian Manufacturing Industries. Regional Science and Urban Economics 51:47-69

Behrens K, Guillain R (2017) The determinants of coagglomeration: Evidence from functional employment patterns. Discussion Paper 11884, CEPR - Centre for Economic Policy Research

Billings SB, Johnson EB (2012) A non-parametric test for industrial specialization. Journal of Urban Economics 71(3):312-331

Bocci C, Rocco E (2016) Modelling the location decisions of manufacturing firms with a spatial point process approach. Journal of Applied Statistics 43(7):1226-1239

Bonneu F (2007) Exploring and Modeling Fire Department Emergencies with a Spatio-Temporal Marked Point Process. Case Studies in Business, Industry and Government Statistics 1(2):139-152

Bonneu F, Thomas-Agnan C (2015) Measuring and Testing Spatial Mass Concentration with Micro-geographic Data. Spatial Economic Analysis 10(3):289-316

Briant A, Combes PP, Lafourcade M (2010) Dots to boxes: Do the size and shape of spatial units jeopardize economic geography estimations? Journal of Urban Economics 67(3):287-302

Brülhart M, Traeger R (2005) An Account of Geographic Concentration Patterns in Europe. Regional Science and Urban Economics 35(6):597-624

Buzard K, Carlino GA, Hunt RM, Carr JK, Smith TE (2017) The agglomeration of American R\&D labs. Journal of Urban Economics 101:14-26

Chain CP, Santos AC, Castro LG, Prado JW (2019) Bibliometric analysis of the quantitative methods applied to the measurement of the industrial clusters. Journal of Economic Surveys 33(1):60-84

Combes PP, Overman HG (2004) The spatial distribution of economic activities in the European Union. In: Henderson JV, Thisse JF (eds) Handbook of Urban and Regional Economics, vol 4, Elsevier. North Holland, Amsterdam, chap 64, pp 2845-2909

Combes PP, Mayer T, Thisse JF (2008) Economic Geography, The Integration of Regions and Nations. Princeton University Press, Princeton

Diggle PJ (1983) Statistical analysis of spatial point patterns. Academic Press, London 
Diggle PJ, Chetwynd AG (1991) Second-Order Analysis of Spatial Clustering for Inhomogeneous Populations. Biometrics 47(3):1155-1163

Dubé J, Brunelle C (2014) Dots to dots: a general methodology to build local indicators using spatial micro-data. The Annals of Regional Science 53(1):245-272

Duranton G (2008) Spatial Economics. In: Durlauf SN, Blume LE (eds) The New Palgrave Dictionary of Economics, Palgrave Macmillan

Duranton G, Overman HG (2005) Testing for Localisation Using Micro-Geographic Data. Review of Economic Studies 72(4):1077-1106

Duranton G, Overman HG (2008) Exploring the Detailed Location Patterns of UK Manufacturing Industries using Microgeographic Data. Journal of Regional Science 48(1):213243

Ellison G, Glaeser EL (1997) Geographic Concentration in U.S. Manufacturing Industries: A Dartboard Approach. Journal of Political Economy 105(5):889-927

Ellison G, Glaeser EL, Kerr WR (2010) What Causes Industry Agglomeration? Evidence from Coagglomeration Patterns. The American Economic Review 100(3):1195-1213

Florence PS (1972) The Logic of British and American Industry: A Realistic Analysis of Economic Structure and Government, 3rd edn. Routledge \& Kegan Paul, London

Gini C (1912) Variabilità e mutabilità, vol 3. Università di Cagliari

Giuliani D, Arbia G, Espa G (2014) Weighting Ripley's KFunction to Account for the Firm Dimension in the Analysis of Spatial Concentration. International Regional Science Review 37(3):251-272

Gómez-Antonio M, Sweeney S (2018) Firm location, interaction, and local characteristics: A case study for madrid's electronics sector. Papers in Regional Science 97(3):663685

Guimarães P, Figueiredo O, Woodward D (2009) Dartboard tests for the location quotient. Regional Science and Urban Economics 39(3):360-364

Henderson JV, Thisse JF (2004) Handbook of Urban and Regional Economics. Elsevier. North Holland, Amsterdam

Howard E, Newman C, Tarp F (2016) Measuring industry coagglomeration and identifying the driving forces. Journal of Economic Geography 16(5):1055-1078

Illian J, Penttinen A, Stoyan H, Stoyan D (2008) Statistical Analysis and Modelling of Spatial Point Patterns. Statistics in Practice, Wiley-Interscience, Chichester
Jensen P, Michel J (2011) Measuring spatial dispersion: exact results on the variance of random spatial distributions. The Annals of Regional Science 47(1):81-110

Kerr WR, Kominers SD (2015) Agglomerative Forces and Cluster Shapes. The Review of Economics and Statistics 97(4):877-899

Koh HJ, Riedel N (2014) Assessing the Localization Pattern of German Manufacturing and Service Industries: A Distancebased Approach. Regional Studies 48(5):823-843

Krugman P (1991) Geography and Trade. MIT Press, London

Law R, Illian J, Burslem D, Gratzer G, Gunatilleke CVS, Gunatilleke I (2009) Ecological information from spatial patterns of plants: insights from point process theory. Journal of Ecology 97(4):616-628

Marcon E, Puech F (2003) Evaluating the Geographic Concentration of Industries Using Distance-Based Methods. Journal of Economic Geography 3(4):409-428

Marcon E, Puech F (2010) Measures of the Geographic Concentration of Industries: Improving Distance-Based Methods. Journal of Economic Geography 10(5):745-762

Marcon E, Puech F (2012) A typology of distance-based measures of spatial concentration. HAL SHS 00679993(version 1)

Marcon E, Puech F (2015) Mesures de la concentration spatiale en espace continu : théorie et applications. Économie et Statistique 474:105-131

Marcon E, Puech F (2017) A typology of distance-based measures of spatial concentration. Regional Science and Urban Economics 62:56-67

Marcon E, Traissac S, Puech F, Lang G (2015) Tools to Characterize Point Patterns: dbmss for R. Journal of Statistical Software 67(3):1-15

Møller J, Waagepetersen RP (2004) Statistical Inference and Simulation for Spatial Point Processes, Monographs on Statistics and Applies Probabilities, vol 100. Chapman and Hall

Mori T, Smith TE (2013) A probabilistic modeling approach to the detection of industrial agglomerations. Journal of Economic Geography 14(3):547-588

Nakajima K, Saito YU, Uesugi I (2012) Measuring economic localization: Evidence from Japanese firm-level data. Journal of the Japanese and International Economies 26(2):201220

Ó hUallacháin B, Leslie TF (2007) Producer Services in the Urban Core and Suburbs of Phoenix, Arizona. Urban Studies 44(8):1581-1601 
Openshaw S, Taylor PJ (1979) A million or so correlation coefficients: three experiments on the modifiable areal unit problem. In: Wrigley N (ed) Statistical Applications in the Spatial Sciences, Pion, London, pp 127-144

Pavia J (2015) Testing goodness-of-fit with the kernel density estimator: Gofkernel. Journal of Statistical Software 66(1):1-27

Penttinen A (2006) Statistics for Marked Point Patterns. In: The Yearbook of the Finnish Statistical Society, The Finnish Statistical Society, Helsinki, pp 70-91

Penttinen A, Stoyan D, Henttonen HM (1992) Marked Point Processes in Forest Statistics. Forest Science 38(4):806824

R Development Core Team (2018) R: A Language and Environment for Statistical Computing

Ripley BD (1976) The Second-Order Analysis of Stationary Point Processes. Journal of Applied Probability 13(2):255266

Ripley BD (1977) Modelling Spatial Patterns. Journal of the Royal Statistical Society B 39(2):172-212

Rosenthal SS, Strange WC (2001) The Determinants of Agglomeration. Journal of Urban Economics 50(2):191-229

Silverman BW (1986) Density estimation for statistics and data analysis. Chapman and Hall, London

Sweeney S, Gómez-Antonio M (2015) Localization and industry clustering econometrics: An assessment of Gibbs models for spatial point processes. Journal of Regional Science 56(2):257-287

Sweeney SH, Feser EJ (1998) Plant Size and Clustering of Manufacturing Activity. Geographical Analysis 30(1):4564

Waller L (2010) Point Process Models and Methods in Spatial Epidemiology. In: Gelfand A, Diggle P, Guttorp P, Fuentes M (eds) Handbook in Spatial Statistics, CRC Handbooks of Modern Statistical Methods Series, Chapman \& Hall, chap 22, pp 403-423

Zhou T, Clapp JM (2015) The location of new anchor stores within metropolitan areas. Regional Science and Urban Economics 50:87-107 